\title{
Professionell handeln - gerade wenn es kritisch wird!
}

\author{
Jürg Schlup \\ Dr. med., Präsident der FMH
}

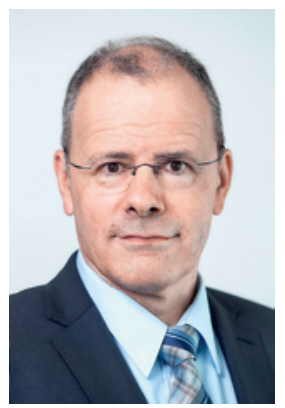

Unser wichtiges Ziel 2019 ist das Einreichen eines mit den Tarifpartnern finalisierten Tarifvorschlags beim Bundesrat - allen Einkommens-Diffamierungen und Staatstarifplänen zum Trotz. Das Stemmen dieser Herausforderung bildet jedoch nur einen Teil unserer anspruchsvollen Aufgaben im Wahljahr, denn 2019 stehen weitere, für die Zukunft unseres Gesundheitswesens entscheidende politische Geschäfte auf der Agenda:

Für Patientinnen und Patienten wie auch für uns Ärztinnen und Ärzte von grösster Tragweite ist das drohende Globalbudget, das uns zum Rationieren nötigen würde, um vorgegebene Kostengrenzen einzuhalten. Ein solches Budget soll aktuell mit dem bundesrätlichen Kostendämpfungspaket 1 weitgehend unbemerkt eingeführt werden (Art. 47c KVG). Die Vernehmlassung endete vor drei Wochen - der weitere Weg dieser Gesetzesrevision in diesem Jahr 2019 bleibt spannend. Gleichzeitig sammelt die CVP bereits Unterschriften für ihre Volksinitiative «Kostenbremse» - ebenfalls ein fixer Kostendeckel -, ohne den Stimmbürgern die konkreten Folgen für die Patientenversorgung aufzuzeigen.

Im Wahljahr 2019 stehen für unser Gesundheitswesen entscheidende Weichenstellungen an.

Ausserdem wird uns 2019 auch die gesetzliche Regelung der Steuerung ambulant tätiger Ärztinnen und Ärzte beschäftigen. Gemäss der vom Nationalrat im Dezember mit grosser Zustimmung beschlossenen Vorlage könnten künftig die Versicherer - nicht mehr die Patienten - die praxisambulanten Ärzte auswählen, die zu Lasten der OKP behandeln dürfen. Dass gleichzeitig die Zulassungssteuerung mit der einheitlichen Finanzierung ambulanter und stationärer Leistungen (EFAS) verknüpft wird, lässt zudem eine Blockade dieser effizienzsteigernden und prämiensparenden Reform befürchten.

Dies ist nur eine kleine Auswahl von anstehenden Weichenstellungen, die den Einsatz der FMH für eine

allgemein zugängliche, qualitativ hochstehende und finanziell tragbare medizinische Versorgung prägen werden. Dabei müssen wir aber nicht mehr nur in sachlichen Auseinandersetzungen bestehen. Gerade das letzte Jahr zeigte, wie Fakten und gute Argumente in aufgeheizten Debatten untergehen können. Für die Ärzteschaft stellt sich daher immer mehr die Frage: Wie können wir unsere Positionen am besten vertreten - in einem Umfeld, das zunehmend von Verkürzungen und Skandalisierungen lebt? Ziehen wir mit sachlichen Argumentationen nicht immer den Kürzeren?

Wir bleiben professionell, um unsere Profession glaubhaft zu vertreten.

Die Antworten der Ärzteschaft und ihrer Organisationen auf diese Fragen sind vielfältig - wie sich am breiten Spektrum ihrer Aktivitäten ablesen lässt. Dennoch orientieren wir uns alle an zentralen gemeinsamen Werten, die uns nicht zuletzt durch unsere Standesordnung vorgegeben sind: Als Ärztinnen und Ärzte sind wir gehalten, unseren Beruf «sorgfältig und gewissenhaft» auszuüben und uns "dadurch des Vertrauens der Ratsuchenden und der Öffentlichkeit würdig» zu erweisen [1]. Dies gilt auch in der Standespolitik: Wir vertreten unseren Berufsstand am besten, wenn wir dem Grundsatz unserer Standesordnung entsprechend "sorgfältig und gewissenhaft» handeln. Dies umfasst auch, unbequeme Wahrheiten auszusprechen und falsche Aussagen richtigzustellen tatsachenbasiert, sachlich und lösungsorientiert. Gerade in kritischen Situationen müssen wir dem Vertrauen, das in unseren Berufsstand gesetzt wird, besonders gerecht werden.

Diesen Herausforderungen werden wir uns auch im Wahljahr 2019 stellen: für ein gutes Gesundheitswesen eintreten - entschlossen, glaubhaft und professionell.

Literatur

1 Standesordnung der FMH. URL: https://www.fmh.ch/files/pdf21/ Standesordnung_August_2018_D.pdf 